\title{
HUMAN++: Emerging Technology for Body Area Networks
}

\author{
Julien Penders ${ }^{1}$, Bert Gyselinckx ${ }^{1}$, Ruud Vullers ${ }^{1}$, Olivier Rousseaux ${ }^{1}$, Mladen \\ Berekovic $^{1}$, Michael De Nil ${ }^{3}$, Chris Van Hoof ${ }^{2}$, Julien Ryckaert ${ }^{2}$, Refet Firat \\ Yazicioglu $^{2}$, Paolo Fiorini ${ }^{2}$, Vladimir Leonov ${ }^{2}$ \\ ${ }^{1}$ IMEC N1/Holst Centre, High Tech Campus 48, 5656AE Eindhoven, The Netherlands. \\ ${ }^{2}$ IMEC, Kapeldreef 75, B-3001 Leuven, Belgium. \\ ${ }^{3}$ Technische Universiteit Eindhoven, Den Dolech 2, 5612 AZ Eindhoven, The Netherlands.
}

\begin{abstract}
This paper gives an overview of results of the Human++ research program [1]. This research aims to achieve highly miniaturized and nearly autonomous sensor systems that assist our health and comfort. It combines expertise in wireless ultra-low power communications, packaging and 3D integration technologies, MEMS energy scavenging techniques and low-power design techniques.
\end{abstract}

Key words. BAN, microsystem, wireless, autonomous, integration

\section{Introduction}

Many national health services struggle in the face of financial resource constraints and shortages of skilled labor. The cost of healthcare delivery is steadily on an upward trend. A recent survey shows that by 2020, healthcare spending is projected to triple in dollars, consuming $21 \%$ of GDP in the US and $16 \%$ of GDP in other OECD countries. As a result, the pressure on health systems to step up efforts in cost containment and efficiency improvement keeps growing. Consensus about the main determinants of expenditure is not complete but revolves generally around cost drivers such as rising income and patient expectations; demographic change, in particular the ageing of population; and new technologies.

In wealthier nations consumer demand increases, leading to a higher spend on healthcare. Statistics show that the cost lowering effect of technology and automation is more than offset by the impact of an ageing society, consumerism, biotechnology and medical breakthroughs. This results in an overall increase in cost between $2-3 \%$ per year. As a result, alternative ways of increasing efficiency, productivity and usability while controlling cost are being sought. One strategy that is gaining major attention consists of offloading healthcare institutions by shifting the health management outside the expensive formal medical institutions. Other strategies seek to improve the appropriateness of treatment or emphasize preventive care rather than treatment. For example, the field of chronic diseases is a vast domain in which the provision of

Please use the following format when citing this chapter:

Penders, J., Gyselinckx, B., Vullers, R., Rousseaux, O., Berekovic, M., De Nil, M., Van Hoof, C., Ryckaert, J., Yazicioglu, R.F., Fiorini, P. and Leonov, V., 2007, in IFIP International Federation for Information Processing, Volume 249, VLSI-SoC: Research Trends in VLSI and Systems on Chip, eds. De Micheli, G., Mir, S., Reis, R., (Boston: Springer), pp. 377-397 
real-time data from and to the patient anywhere and at any point in time may hold significant potential for cost reduction.

The supporting role of an adequate technology platform is critical here. E-health technology, enabling wireless and mobile based healthcare services, is increasingly coined as the revolutionizing enabler for the next decades to come. As defined by the WHO, e-health refers to the use in the health sector, of digital data transmitted, stored and retrieved electronically for clinical, educational and administrative purposes, both at the local site and at a distance. There are three strong drivers for an e-Health technology platform. The first, demographic, is the evolution toward an ageing society, active ageing and independent living. This calls for radical changes in how care will be provided for the elderly and how technology may assist. Second is the epidemiologic transition from episodic to chronic healthcare needs. Future healthcare systems should thus focus on prevention, on effective provision of continuous treatment, on integrating lifestyle parameters, and should be customized to individual needs of each patient. Finally, patients expect ever more from health services, reinforcing the existing concept of a 'patient centric view' of healthcare which emphasizes the patient's experience and journey through a system that provides continuity of care to a proactive patient.

E-health is claimed to offer the potential to reduce costs, enable personalized healthcare, deliver remote health services and increase the delivery efficiency in realtime. However, at this early stage today it is only through the many pilot projects on e-health ongoing in different countries around the world that evidence will be gathered to determine the economic viability, and answer the question how e-health can enhance the healthcare system efficiency and resolve the associated cost burden.

\section{An Enabling Technology: Body Area Networks}

In this text we analyze one component of e-health, the personal body area network (BAN) [2] that provides medical, lifestyle, assisted living, sports or entertainment functions for the user. This network comprises a series of miniature sensor/actuator nodes each of which has its own energy supply, consisting of storage and energy scavenging devices. Each node has enough intelligence to carry out its task. Each node is able to communicate with other sensor nodes or with a central node worn on the body. The central node communicates with the outside world using a standard telecommunication infrastructure such as a wireless local area or cellular phone network. Experts might then provide services to the individual wearing the BAN, such as management of chronic disease, medical diagnostic, home monitoring, biometrics, and sport and fitness tracking. Next generation of BAN will include feedback loops for disease management or drug and treatment delivery within so-called closed-loop systems, and will provide feedback to the individual about her lifestyle and health status, eventually leading to human-in-the-loop systems. 


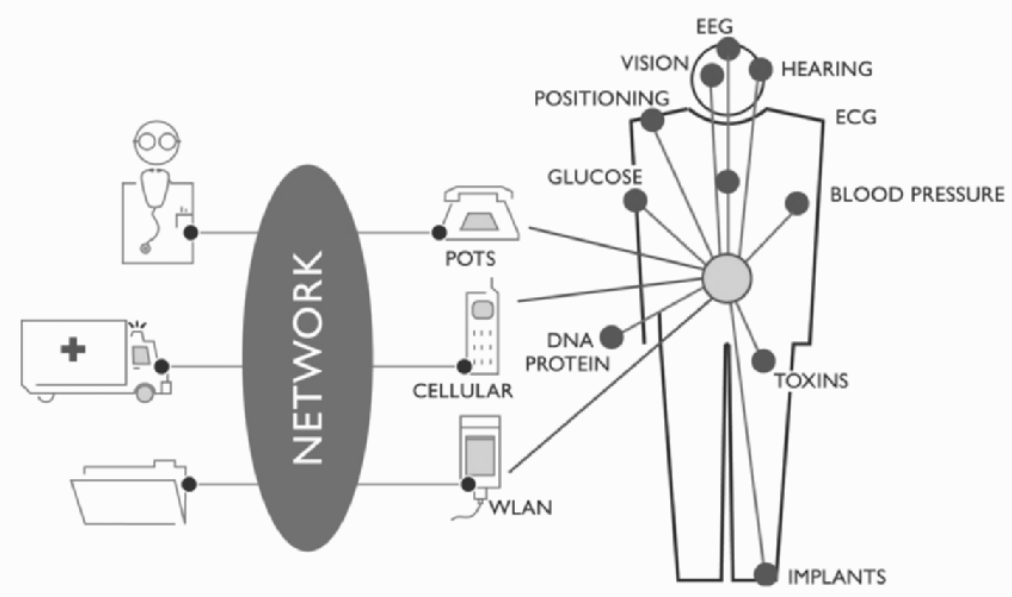

Fig. 1. The technology vision for the year 2010: people will be carrying their personal body area network and be connected with service providers regarding medical, lifestyle, assisted living, sports and entertainment functions.

The successful realization of this vision requires innovative solutions to remove the critical technological obstacles. First, the overall size should be compatible with the required form factor. This requires new integration and packaging technologies. Second, the energy autonomy of current battery-powered devices is limited and must be extended. Further, interaction between sensors and actuators should be enlarged to enable new applications such as multi-parameter biometrics or closed loop disease management systems. Next, intelligence should be added to the device at the node level so that each node is capable of storing, processing and transferring data continuously or on an event-triggered basis. Intelligence should also be introduced at the network level to deal with issues such as network management, data integration and data interpretation. Finally, the energy consumption of all building blocks needs to be drastically reduced to allow energy autonomy.

The Human++ program is looking into all of these generic BAN challenges. In the following sections we will have a closer look at the technologies under development. We will start with an overview of the test case, then we will discuss the enabling technologies such as wireless communication, micropower generation, digital signal processing and sensors. Finally, we show how advanced integration technology can bring together all the heterogeneous subcomponents in a compact form factor.

\section{Ambulatory Multi-Parameter Monitoring as Test Case}

We selected ambulatory multi-parameter monitoring as a driving application for Human++. The target of such a monitoring system is to acquire process, store, and visualize a number of physiological parameters in an unobtrusive way. In one case, 
we focused on the simultaneous acquisition of EEG/ECG/EMG biopotential signals. Traditionally, such signals are either captured in a clinical setting for immediate interpretation or they are recorded in an ambulant setting for post factum analysis via a Holter monitor. With a wireless ambulatory monitoring system we want to combine the real time features of the clinical system with the benefits of ambulatory monitoring from a Holter monitor. Fig. 2 shows a schematic drawing of typical set-up. The set-up consists of:

- 1 EEG sensor node that can acquire, process and transmit 1 to 24 EEG signals;

- 1 ECG sensor node that can acquire, process and transmit ECG signals;

- 1 EMG sensor node that can acquire, process and transmit EMG signals;

- 1 base-station that collects the information from the 3 sensor nodes.

All the sensors have very similar functionality. First, the incoming signals are amplified and filtered. The resulting signals are sampled at $1024 \mathrm{~Hz}$ with a 12-bit resolution. If required, the bio-signals are then processed locally to extract relevant features, e.g. heart rate, energy expenditure or force. Finally, the digital signals are transmitted over a wireless link operating in the $2.4 \mathrm{GHz}$ ISM band. Because the sensors are very similar, they can be realized with the same programmable hardware, illustrated on Fig. 3.

The base-station acts as a data collector. The collected data are passed on to a PC or PDA through a USB interface. Further, the base-station also acts as a master node for the network, which manages the data-flow through the network. BSN are typical star topology networks, for which time division multiple access (TDMA) schemes are well suited.

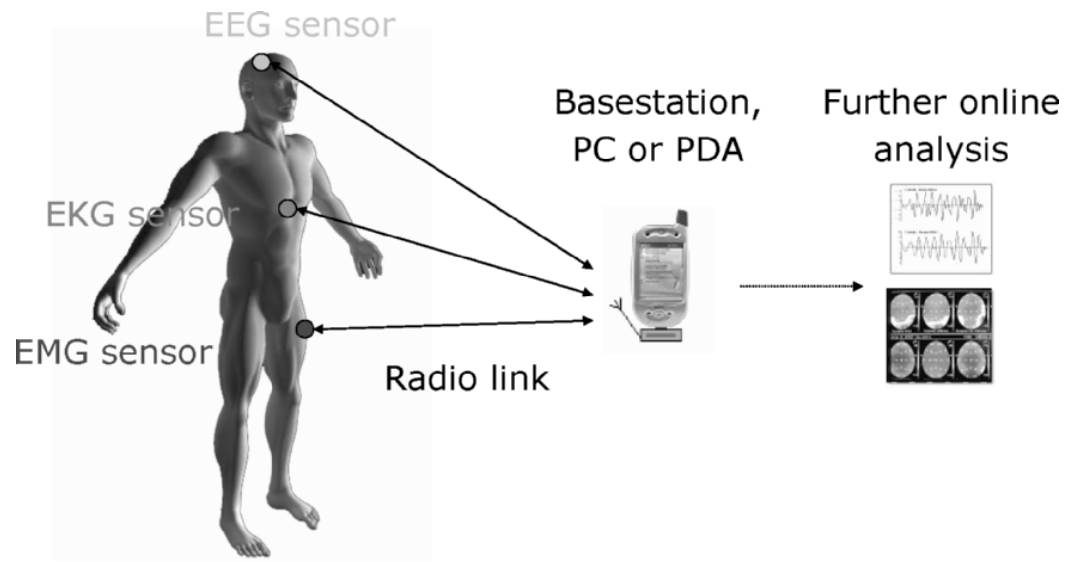

Fig. 2. Schematic overview of the BAN set up, consisting of EEG/ECG/EMG sensors wirelessly connected to a PC or PDA. 

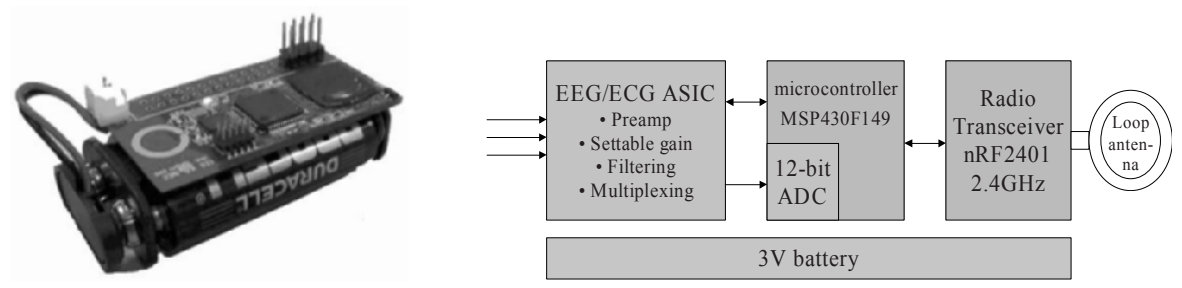

Fig. 3. Close up of the sensor node. On the left a picture and on the right a functional diagram.

A key design criterion for such system is the power of the sensor nodes because this will directly determine the size and the operational lifetime of the system. Analysis of the operation of the sensors shows that they are alternating between four different modes of operation:

1. Listen: the sensors receive their parameters from the base station;

2. Processing: the biopotential signals are monitored and processed;

3. Transmit: the sensors send their data to the base station;

4. Sleep: power save mode - most of the electronics are switched off.

The time spent in each of these modes is very much application dependent. As an example, Fig. 4(a) shows the relative time spent in each of the modes for a particular EMG measurement. In this particular case, signal processing consists of RMS computation. It is clear that for this sensor the idle time is very important and that the system needs to have a very low stand by power consumption.

Each of these modes has its own power consumption. Fig. 4(b) shows that the current consumption in listen and transmit mode is much higher than in processing or sleep mode. This is a direct consequence of the radio which is switched on in these modes and which consumes about $90 \%$ of the power when it is active. Bringing all of these data together we get to the total average power consumption for the sensor: with the current system consisting mainly of off the shelf components a prototype can be designed that consumes less than $1 \mathrm{~mW}$ of power if the measurement interval is longer than $1 \mathrm{~s}$. If we assume that we use two AA batteries in series with a capacity of $2500 \mathrm{mAh}$, the battery lifetime becomes approximately 3 months.
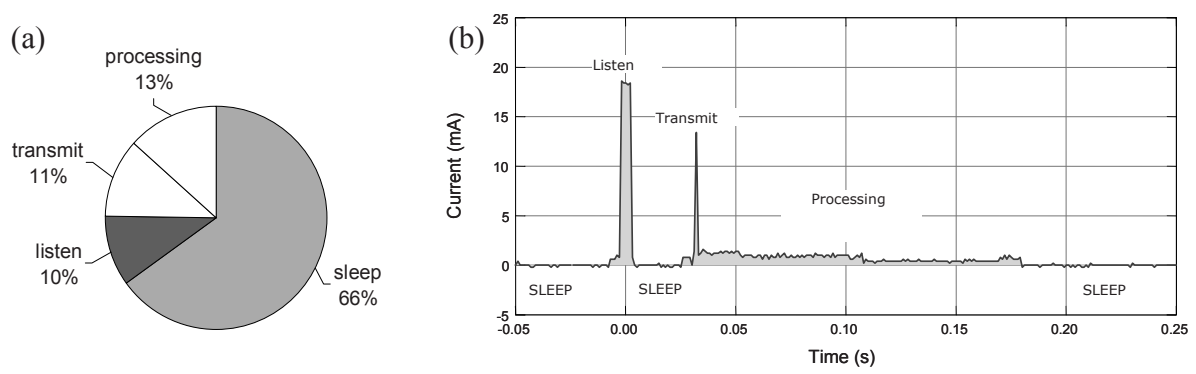

Fig. 4. (a) Relative time spent in different operation modes for a EMG sensor node performing RMS computing locally; (b) Current consumption of sensor node in different operating modes. 
This clearly shows that with today's technology, first realistic demonstrators with a reasonable lifetime can be manufactured. However a couple of major challenges still have to be solved in order to come to a widespread deployment of BANs.

- Miniaturization: in most of current systems, batteries are the single largest contributor to the sensor node size. AA batteries are good for demonstration, but one would like to work with a coin or planar type of battery. These batteries have roughly 100 times less capacity than the AA cells. To keep the same battery lifetime the power of the electronics has to be reduced by a factor 100. Furthermore, development of advanced integration technology is required to achieve compact, flat and flexible sensor nodes, for embodiment in textile or clothing accessories. Making BSN invisible and non-intrusive is likely to determine their acceptance as a support for personalized healthcare and independent living.

- Autonomous systems: the system we demonstrated can run for months. However, to come to a truly autonomous system it should be able to operate over its full lifetime without maintenance. At a given battery capacity, lifetime can be increased by reducing the power of electronics. Alternatively, one can scavenge energy from the environment during the operation of the system. If the average scavenged energy is larger than the average consumed energy, the system can run eternally with the battery or a super capacitor acting only as a temporary energy buffer. A combination of these technologies appears as the optimal solution for achieving autonomous BSN.

- Integration of novel sensor and actuator concepts: the quality of the information resulting from a BAN is only as good as what you measure. Today, often only simple physical properties are measured such as bio-potentials, temperature and movement. Sensors that can measure these parameters in an ambulatory setting are much needed. Motion artifacts are often a major source of data corruption. Implantable sensors face additional issues such as biocompatibility. Besides the monitoring of vital signs and biomedical variables, additional sensors are required for sensing the environment, thus making the system "context aware". This set of heterogeneous sensors need to be secure and reliable. Moreover interference issues need to be addressed, especially for actuating systems where interferences might affect not only the monitoring but also the active part of the system.

- Providing more functionality: most of today's devices act as simple gateways, passing on the information to a central hub where the data is converted into actionable information. By adding intelligence to the sensors they can take decisions locally and the signaling overhead in terms of data and latencies can be reduced. Additionally, intelligence is required for the system to make decisions depending on the status of the environment, thus enabling context-awareness. Finally, embedded intelligence opens the door to closed-loop systems providing action or feedback to the user.

- Become manufacturable at low cost: today's systems cost around 100+ euros. A major reason for this is the low volume of the market so far, but another more technical reason is that there are no commercially available packaging technologies that can efficiently integrate such heterogeneous components as batteries, MEMS, processors, and radios in a single package. 
In the remainder of this chapter we will show how advances in wireless communication, energy scavenging, sensors and system integration can enable such systems in the near future. The hunt for sensor systems that are $1000 \mathrm{x}$ more power efficient, that have ample intelligence to make decisions, that cost less than $1 €$ and that are unobtrusive is open.

\section{Wireless Communication}

Commercially available low power radios, which typically rely on Bluetooth, Zigbee [3] or other proprietary narrowband communication schemes (e.g. Nordic [21]) cannot meet the stringent Wireless BAN power requirements that we are looking for. Typical chipsets for these radios consume in the order of 10 to $100 \mathrm{~mW}$ for data rates of 100 to $1000 \mathrm{kbps}$, leading to a power efficiency of roughly 100 to $1000 \mathrm{~mW} / \mathrm{Mbps}$ or $\mathrm{nJ} / \mathrm{bit}$. Some recent research prototypes or commercial chipsets with limited functionalities have shown efficiencies of $20 \mathrm{~nJ} /$ bit or slightly below [6][7][8]. As outlined in the previous section, we need a radio which is 1 to 2 orders of magnitude more power efficient.

A classical approach to reduce power consumption consists in data-level dutycycling: sensor data are collected in a buffer and organized in packets periodically transmitted by the radio in short communication bursts. If the radio is switched off between communication bursts, this in turn reduces radio duty cycle and therefore reduces power consumption. However, none of the off-the-shelf low-power radio, even duty-cycled, have proven the ability to reduce the power consumption by the required orders of magnitude while offering the necessary communication performances.

Novel air interfaces relying on impulse radio ultra wideband (UWB-IR) radio signals show a strong potential for further reduction of the wireless communications power budget and are currently attracting a lot of attention for the development of next-generation ultra low power radios. In the rest of this section, we highlight how the use of UWB-IR signals allows reducing the power consumption of the radio and then giving an overview of recent achievements in the development and implementation of such systems.

\subsection{UWB air interface: promising candidate for ultra low power wireless communications}

UWB signals are formally defined as radio signals that have a bandwidth larger than $500 \mathrm{MHz}$. The Federal Communications Commission (FCC) has recently authorized UWB communications between $3.1 \mathrm{GHz}$ and $10.6 \mathrm{GHz}$. The regulations on UWB radiation define a power spectral density (PSD) limit of $-41 \mathrm{dBm} / \mathrm{MHz}$, but there are no specific regulations on the definition of the time-domain waveform and there are various ways of obtaining an UWB waveform. In UWB-IR systems, the transmitted signal consists in pulses of short duration ( $\sim 1-2 \mathrm{~ns})$ that are separated by longer silent periods ( $20 \mathrm{~ns}$ or more) as illustrated in Fig. 5. 
(a)

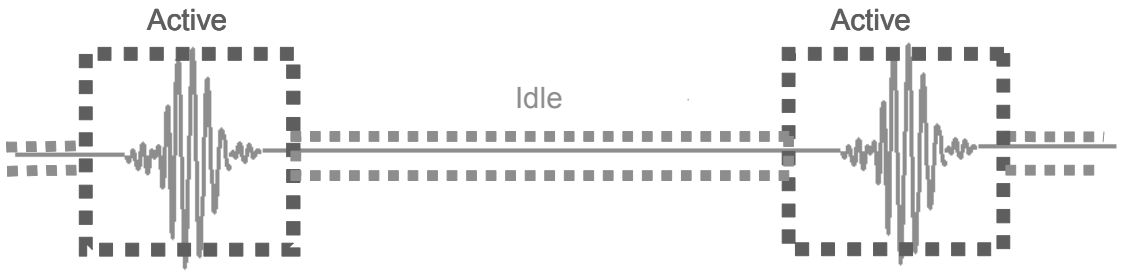

(b)

Active

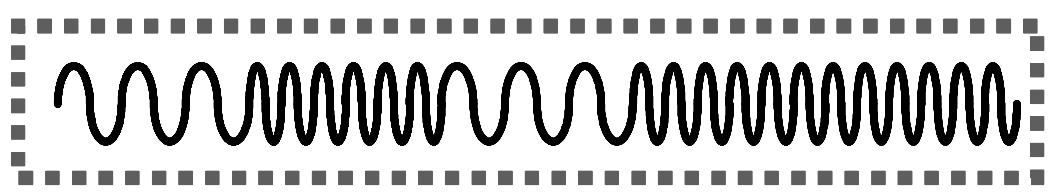

Fig. 5. (a) Schematic representation of a UWB-IR signal allowing signal-level duty cycling; (b) schematic representation of a narrowband radio signal.

Power consumption of UWB-IR systems can be reduced using signal duty cycling, i.e. duty cycling of the radio front-end during data transmission. Indeed, the structure of UWB-IR signals allows to switch off the radio front-end during the silent time intervals between UWB pulses, and to re-activate it only when a pulse needs to be generated, leading to a duty cycle ranging from $1 \%$ to $10 \%$ of the actual data transmission time. Combining duty cycling at signal and data level duty cycling potentially enables effective global duty cycles ranging from $0.1 \%$ to $0.01 \%$. Such a signal duty-cycling approach is not an option in narrowband systems where a continuous waveform must be generated to transmit the data, requiring the front-end to stay in an active mode throughout the actual data transmission interval, as illustrated in Fig. 5.

\subsection{Low Power UWB pulse generator}

In order to achieve ultra-low power consumption, the circuits used for the generation of UWB pulses should have very short start-up times (i.e. a few nanoseconds) to enable efficient signal duty cycling. Furthermore, although no specific waveform is mentioned in international regulations, various UWB standard proposals ([5], [11]) have subdivided the entire UWB spectrum in $500 \mathrm{MHz}$ sub-bands as a solution to compensate for strong interferers, to improve multiple accesses and to compose with the different regulations on UWB emissions worldwide. The emerging 802.15.4a lowpower UWB-IR standard [11] even imposes time-domain specifications for the generated pulses. In order to comply with these regulations and standards, the generated pulses of UWB impulse-radio (UWB-IR) approaches must fulfill stringent spectral masks that can feature such low bandwidths. This poses a serious challenge for the pulse generation of UWB-IR transmitters. 
A low-power and low-complexity implementation of a pulse generator complying with these specifications is proposed in [13]. The proposed pulser architecture generates a triangular baseband pulse shape which is then multiplied with an RF carrier for up-conversion, an approach which is often referred to as carrier-based $U W B$ impulse radio. The triangular waveform is in line with the standard recommendations both in terms of time-domain and spectral specifications with a side-lobe rejection of more than $20 \mathrm{~dB}$. The pulse generator architecture is presented in Fig. 6. A triangular pulse generator and a ring oscillator are activated simultaneously. The choice of a ring type of oscillator is motivated by its fast startup time that enables heavy signal duty-cycling. A gating circuit (ring activation circuit in Fig. 6) manages the signal duty cycling and activates the ring oscillator when a pulse must be transmitted, avoiding wasting power between the pulses.
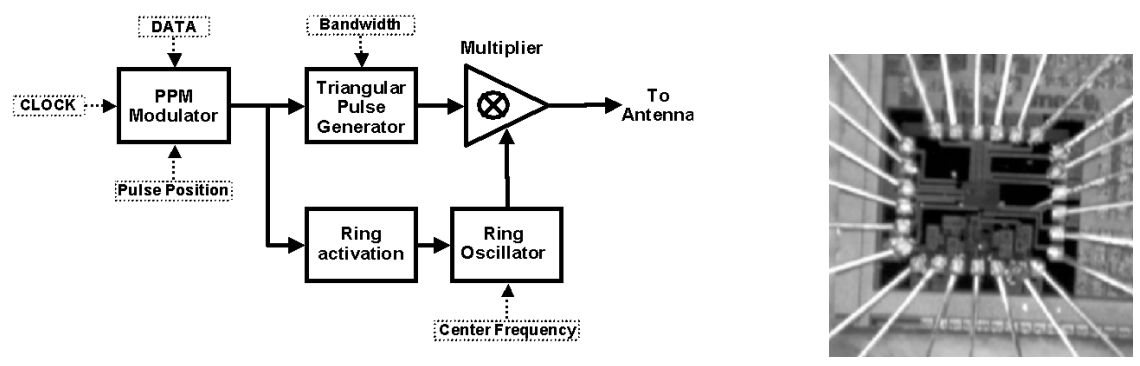

Fig. 6. Architecture of the pulse generator (left) and micrograph of the pulser die (right).

The pulser circuit has been implemented in a logic $0.18 \mu \mathrm{m}$ CMOS technology [14]. The system can deliver a pulse rate up to $40 \mathrm{MHz}$ with a measured energy consumption of $2 \mathrm{~mW}$ or $50 \mathrm{pJ}$ per pulse at a $40 \mathrm{MHz}$ pulse repetition rate. In a typical scenario where one bit of information is encoded over 20 pulses, this represents a power efficiency of roughly $1 \mathrm{~nJ} / \mathrm{bit}$. This is exactly the type of breakthrough low power consumption we were hoping to find.

\subsection{UWB analog receiver}

Processing wideband analog signals in the digital domain requires an extremely fast sampling ADC with a wide input bandwidth. Such solutions exist [15] but usually exhibit high power consumption. In order to minimize the overall sampling rate and total power consumption, analog preprocessing is an interesting alternative. Fig. 7 illustrates an analog-based correlation receiver architecture, which corresponds to a direct down-conversion architecture with an analog quadrature correlator. UWB signals are first down-converted in quadrature baseband, then correlated with a rectangular pulse template. The output of the analog baseband is then sampled at the pulse repetition frequency. The choice of such a simple receive template is driven by the simplicity of its implementation and the consequent low power consumption. 

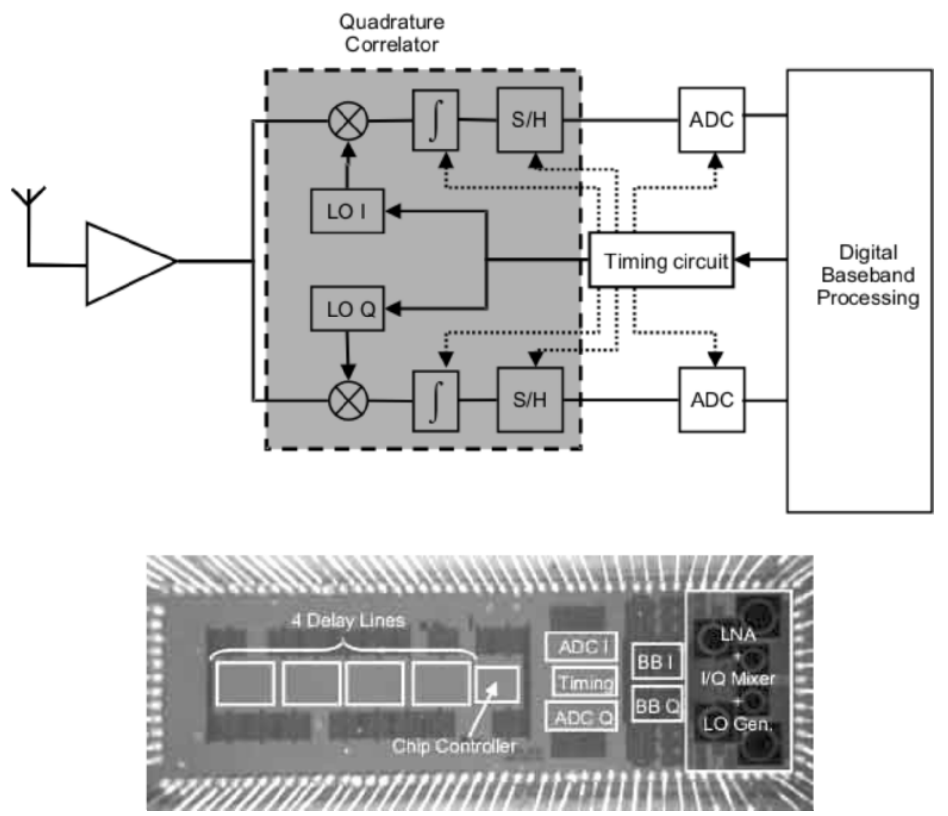

Fig. 7. UWB analog receiver: schematics and chip micrograph.

This receiver has been implemented on a $0.18 \mu \mathrm{m}$ CMOS technology [16]. The total power consumption of the chip is $30 \mathrm{~mW}$ at $20 \mathrm{MHz}$ pulse repetition frequency. This power consumption is more than one order of magnitude higher than for the pulser, and corresponds to an energy efficiency of approx. $10 \mathrm{~nJ} / \mathrm{bit}$. The reason behind this increased power consumption is that signal duty cycling could not be applied in the receiver, since the startup time of some receiver components is larger than the average inter-pulse interval.

The proposed transmitter outperforms low-power radios by more than one order of magnitude, while the receiver has an energy efficiency that compares to narrowband receivers. This asymmetric power consumption is adequate for BAN applications since the most power-constrained elements are the sensors, while the receiver in the central station has slightly more relaxed power budget.

\section{Micropower Generation and Storage}

Today, the batteries that are needed to power wireless autonomous transducer systems seriously limit the possibilities of this emerging technology. Modern electronic components become smaller and smaller, while the scaling of electrochemical batteries faces technological restrictions. As a consequence, either large batteries are used that give a longer autonomy but make the system bigger, or small batteries are used that make the system less autonomous. For this reason, a worldwide effort is ongoing to 
replace batteries with more efficient, miniaturized power sources. We aim at generating and storing power at the micro scale to improve the autonomy or reduce the size of wireless autonomous transducer systems. The envisaged solution takes its energy thermal or mechanical - from the human body and converts it into electrical energy, stored in a micro-battery or (super)capacitor. The choice of scavenging principle depends on the application and the environment in which it is used. In this section, we will present thermal scavengers because they are well suited to convert the thermal body heat into electricity.

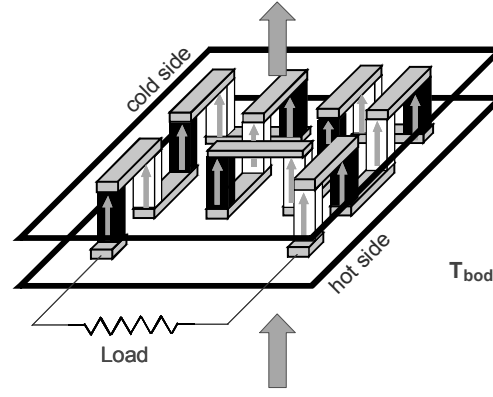

(a)

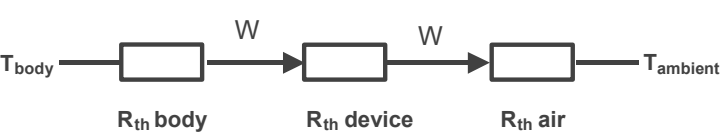

(b)

Fig. 8. (a) Schematic of a thermoelectric generator. (b) The schematic thermal circuit representing the generator and its environment.

Thermal energy scavengers are thermoelectric generators which exploit the Seebeck effect to transform the temperature difference between the environment and the human body into electrical energy. A thermoelectric generator is made of thermopiles, which are in turn made of a large number of thermocouples connected thermally in parallel and electrically in series, as shown schematically in Fig. 8(a). The black and white pillars represent the two types of thermoelectric materials, and the metal interconnects are drawn in grey. They are sandwiched between two plates, the cold and hot sides of the device. Heat flows from the hot side to the cold side, through the pillars (indicated by the arrows). The maximum electrical power is generated when the two following conditions are met: (i) the load is matched to the electrical resistance of the generator and (ii) the thermal conductance of the thermocouples equals the one of the air between the plates. Under this condition, power increases when increasing the height of the pillars.

\subsection{Thermoelectric generators and systems with commercial thermopiles}

A number of TEGs has been built during the last few years. First generation TEGs were introduced to measure the heat flows on man and animals. Although they offer a good power output $(0.1 \mathrm{~mW})$, they usually produce less than $0.5 \mathrm{~V}$ output on the matched load. The second generation of TEGs is characterized by a voltage of more than $0.7 \mathrm{~V}$ which is enough for its effective utilization for powering electronic modules [22]. In 2005, the size of TEGs has been reduced to the one of a man watch thereby significantly improving their acceptance by the wearer [23]. These third 
generation TEGS are intended for use at a temperature of about $22^{\circ} \mathrm{C}$, then providing a useful power of 0.2-0.3 $\mathrm{mW}$ on office workers and about $0.1 \mathrm{~mW}$ at night or e.g. on immobilized patients in hospitals (low metabolic rate), as represented on Fig. 9(a). Therefore, taking into account adverse illumination conditions at home, on transport and at night, the TEGs turn out to be much more powerful than the best solar cells [25].

Integration of thermopiles within sensor modules requires advanced power management schemes to optimize harvested power efficiency. Typically, the TEG continuously charges a battery or a supercapacitor, which then provides power to electronic modules. Voltage up-converters are usually added to match the need for higher voltage power-supply of different electronic components. However, since such converters significantly decrease the overall power efficiency, attention should be paid to match input-voltage of electronic components with the output-voltage of the battery/supercapacitor. In systems exploiting the last generation of TEGs, supercapacitors were preferred to batteries because they can start storing energy at a lower voltage. At a given temperature, this means more energy can eventually be stored; at a given power need, this means the device could work at higher air-temperature.

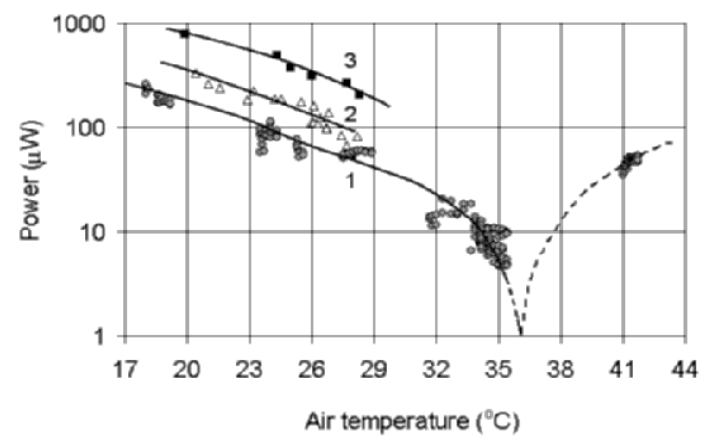

Fig. 9. Performance characteristics of the watch-like TEG. Power vs. air temperature produced at different metabolic rate levels: on a walking man (3), when working in office (2), and with no physical activity for prolonged period (1).

In 2006, we achieved the development of the first prototype of a non-trivial biomedical sensor fully powered by the patient's body heat: a wireless pulse oximeter for non-invasive measurement of pulse and blood oxygen [24], shown in Fig. 10. The device consists of the watch-size wrist TEG, sensor electronics, processor and radio, and a commercial transmission pulse oximetry finger sensor. The watch-size wrist TEG is connected to a supercapacitor as described above. For digital signal processing and radio communication, the system uses the wireless sensor platform presented in the test case. The sensor locally performs all signal processing on $3.9 \mathrm{~s}$ blocks of measurements, and transmits the resulting pulse and oxygen saturation values (Fig. 10). This leads to a sustainable average power consumption of about $100 \mu \mathrm{W}$. 

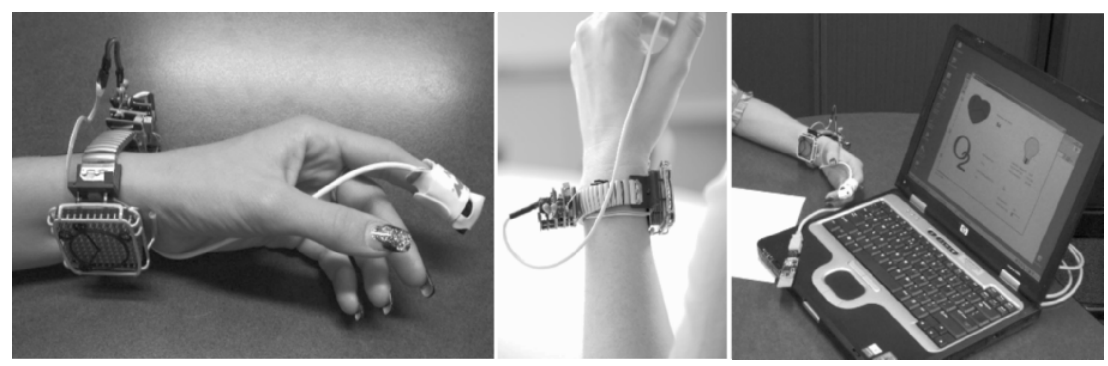

Fig. 10. Wireless body-powered pulse oximeter (left), a prototype of the battery-less electronics (middle) and the application running on a laptop (2006).

\subsection{Micromachined thermopiles}

The use of commercial thermopiles has proven that human heat can be used to power a sensor node. Nevertheless, the solution is non-optimal for two reasons: (i) it does not offer the possibility of optimizing the power and the voltage at the same time, and (ii) it is a very expensive one, because thermopiles fabrication techniques cannot be easily automated. A possible solution could be the use of micromachined thermopiles. These have already been presented in the scientific literature, and are used in miniaturized commercial thermoelectric coolers. Micromachining has the potential advantage of reducing the lateral size of the thermocouple. This means that a much larger number of thermocouples can be fabricated per unit area, thus allowing to matching of thermal conductance of thermopile to the one of the air. Such approach would allow combining a large voltage and a large power. Unfortunately, micromachined thermocouples have a height of a few microns only, which drastically reduces the thermal resistance of the generator and, hence, the generated power.

In order to overcome this difficulty, we have developed a special design of a micromachined thermoelectric generator for application on humans, which combines a large thermal resistance of the device with a large number of thermopiles. The schematic is shown in Fig. 11(a). Several thousands of thermocouples are micromachined on a silicon rim. The function of this rim is to increase the parasitic plateto-plate thermal resistance of the generator. If $\mathrm{Bi}_{2} \mathrm{Te}_{3}$ is used as thermoelectric material an optimized device fabricated according to this scheme and positioned on the human wrist can generate up to $30 \mu \mathrm{W} / \mathrm{cm}^{2}$ at a voltage exceeding 4 volts. Fig. 11(c) shows a realization of such a device based on SiGe thermocouples. Because of the inferior thermoelectric properties of this material with respect to $\mathrm{Bi}_{2} \mathrm{Te}_{3}$, an optimized device is expected to generate about $5 \mu \mathrm{W} / \mathrm{cm}^{2}$ at a voltage of 1.5 Volts. 


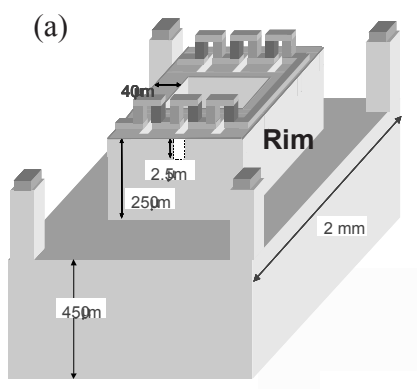

(c)

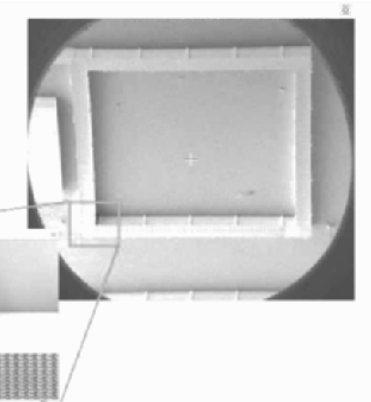

(b)

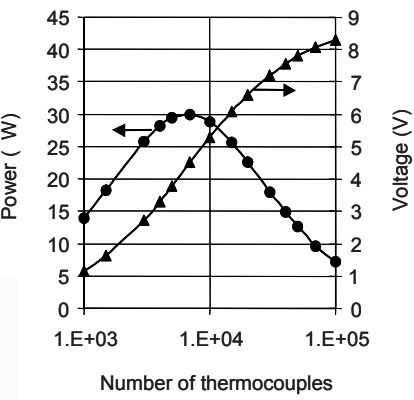

Fig. 11. (a) Schematic of the TEG capable of combining large power and large voltage (rim and thermopiles are not scaled to overall device dimensions). (b) Simulated performance of the TEG shown in (a). (c) Photograph of the fabricated thermoelectric generator. Thermocouples are made of SiGe.

\section{Digital Signal Processing}

The ambulatory monitoring test-case has shown that the large majority of power is spent on the wireless transmission of the data (see Fig. 4(a)). As discussed, UWB communication is foreseen as an efficient way to reduce power consumption due to wireless communication. For most applications, further reduction can be obtained by performing local signal processing on the node, hence avoiding the transmission of the entire raw data (bandwidth reduction). Technologies to enable local ultra-lowpower signal processing should thus be considered. Among these, Digital Signal Processors (DSPs) are particularly well-suited for tight power budget, while still offering a decent level of flexibility. Here we discuss the development of Ultra Low Power (ULP) Digital Signal Processors for BSN, which shall be capable of running on scavenged energy only.

As the intensity of requested local processing is highly dependent on the application, we will illustrate our developments on the particular case of ECG monitoring, part of the ambulatory multi-parameter monitoring test-case. An extensive overview of algorithms available for ECG analysis and QRS detection can be found in [26]. We illustrate our developments on an algorithm for R-peak detection described in [28]. Since only R-peak information is then transmitted through the radio, this typically reduces the data-rate by a factor 1000 (from $10+\mathrm{kbps}$ to $10+\mathrm{bps}$ ), thus resulting in a better balance between computation and communication. 
The typical power breakdown of a (DSP) microprocessor is given by:

- Leakage Power $\left(\mathrm{P}_{\text {leak }}\right)$ : static dissipation while the processor is powered on.

- Active Power $\left(\mathrm{P}_{\text {active }}\right)$ : dynamic dissipation consumed while the processor is active.

- Idle Power $\left(\mathrm{P}_{\text {idle }}\right)$ : dynamic dissipation consumed while the processor is in stand-by mode without processing data.

Total power consumption is thus given by:

$$
P_{\text {Total }}=P_{\text {Leakage }}+f_{\text {sample }} *\left(\left(P_{\text {Active }} \cdot t_{\text {Active_avg }}\right)+\left(P_{\text {Idle }} \cdot t_{\text {Idle_avg }}\right)\right),
$$

where $f_{\text {sample }}$ is the sample rate of the incoming data, $t_{\text {active, avg }}$ the average time needed for processing one sample and $t_{\text {idle, avg }}$ the average time the processor is idle after processing a sample. Both $t_{\text {active,avg }}$ and $t_{\text {idle,avg }}$ depend on the processor clock speed with $1 / f_{\text {sample }}=\left(\mathrm{t}_{\text {active,avg }}+\mathrm{t}_{\mathrm{idle} \text {,avg }}\right)$. Also $\mathrm{P}_{\text {active }}$ depends on the processor clock speed with $\left(P_{\text {active }} * t_{\text {active, avg }}\right)=$ energy/sample, constant for every active operation.

Simplistic approaches to achieving low power performance consist in reducing processor clock frequency to its minimal value, $\mathrm{f}_{\text {sample }} * \max (\mathrm{cycles})$, where $\max$ (cycles) is the maximum number of cycles required to process a data sample. This technique can be applied to any processors and results in a reduction in idle time and active power while, however, increasing processor response time. Alternatively, more advanced optimization techniques can be used to achieve ultra low power performances. They fall under three main categories:

- Idle power is reduced by implementing a top level clock gating mechanism, on top of the existing fine-grain clock-gating, which eliminates any internal switching activity, thus reducing internal idle switching energy to zero.

- Active power reduction is achieved by decreasing the amount of execution cycles and optimizing the signal processing algorithm using embedded computing techniques including conversion from floating- to fixed-points, and implementation of approximations for divisions, trigonometric and other complex mathematical functions.

- Leakage power consumption is cut down by using high threshold voltage $\left(\right.$ High- $\mathrm{V}_{\mathrm{T}}$ ) libraries for standard cells and memories.

- $\quad$ Scaling down program memory from 128 to 64 bits, reducing data memory sizes and decreasing processor complexity.

We emphasize the impact of these optimizations on a benchmarking example, in which we mapped the R-peak detection algorithm onto a processor using the coarsegrained reconfigurable technology of Silicon Hive [27]. The algorithm executes on the Silicon Hive processor at $100 \mathrm{MHz}$ with a sample rate of $200 \mathrm{~Hz}$. The empirical results obtained before optimization suggested a duty cycle of $0.25 \%$, showing that most of the power is consumed when the processor is idle. Dynamic and static optimization of power consumption led to the following results: 
- Dynamic idle power consumption is reduced to $\sim 0 \mu \mathrm{W}$ (clock consumption).

- Cycle count is reduced by a factor of five, therefore driving active time down to only $0.05 \%$.

- Leakage power is decreased by a factor 14 , mainly thanks to the use of high threshold voltage (factor 7).

Power analysis after a gate-level simulation, with wire-capacitances back-annotated from layout, reports a power consumption of $7.81 \mu \mathrm{W}(5.45 \mu \mathrm{W}$ leakage, $2.36 \mu \mathrm{W}$ active). Table 1 summarizes the power optimization achieved for our benchmarking case, compared to an initial Silicon Hive (generic purpose RISC) processor running at $100 \mathrm{MHz}$ and at $1.25 \mathrm{MHz}$, minimal clock frequency in this case. Further reduction of leakage power during idle time could be achieved by dynamic power switching or dynamic voltage scaling. Since the processor spends most of its time in idle mode (Table 1), this should lead to a leakage power below $1 \mu \mathrm{W}$.

This discussion has shown that Digital Signal Processors, combining both flexibility and efficiency, are suitable for BSN applications powered by energy scavenging technology. Future research should focus on additional architectural explorations, application analysis and advanced power optimizations e.g. power gating and data path enhancement.

Table 1. Power breakdown for benchmarking example (see text).

\begin{tabular}{|l|c|c|c|}
\hline & $\begin{array}{c}\text { Original @ } \\
\text { 100Mhz }\end{array}$ & Original @ 1.25Mhz & $\begin{array}{c}\text { Optimized @ } \\
100 \mathrm{Mhz}\end{array}$ \\
\hline Active & $6.52 \mathrm{~mW} \star 0.25 \%$ & $81.55 \mu \mathrm{W} * 20.00 \%$ & $4.71 \mathrm{~mW} * 0.05 \%$ \\
\hline Idle & $0.75 \mathrm{~mW} \star 99.75 \%$ & $9.41 \mu \mathrm{H} * 80.00 \%$ & $0.00 \mathrm{~mW} * 99.95 \%$ \\
\hline Leakage & $100.04 \mu \mathrm{W}$ & $100.04 \mu \mathrm{W}$ & $5.45 \mu \mathrm{W}$ \\
\hline TOTAL P & $\mathbf{8 6 7 . 4 7 \mu W}$ & $\mathbf{1 2 3 . 8 4 \mu W}$ & $\mathbf{7 . 8 1} \mu \mathrm{W}$ \\
\hline
\end{tabular}

Acknowledgments. Results presented in this section were obtained in collaboration with Silicon Hive, the TU Eindhoven and Philips Research. The authors would like to thank Frank Bouwens, Lennart Yseboodt, Jos Huisken and Jef van Meerbergen for their contribution.

\section{Sensors and Actuators}

Our multi-parameter monitoring system needs to acquire biopotential signals in a very power efficient way. The different biopotentials have different amplitude and frequency characteristics as shown in Fig. 12. For this purpose, we developed a low-power 25 channel biopotential ASIC [30]. The ASIC preprocesses typical biopotentials such as ECG and EEG signals. It can be configured in different operational modes thanks to its variable bandwidth and gain settings. 


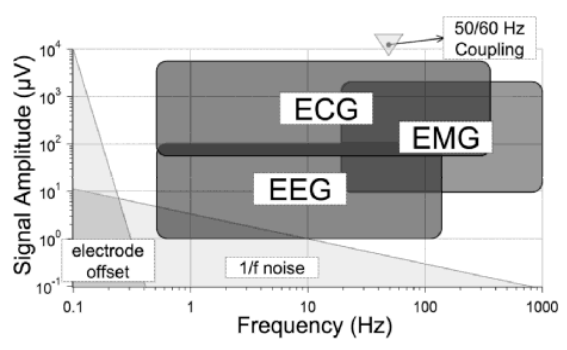

Fig. 12. Amplitude and frequency characteristics of different biopotential signals.

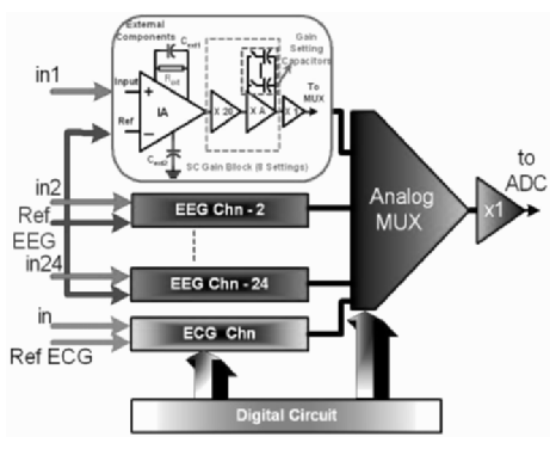

Fig. 13. 25 channel biopotential ASIC.

The mixed-signal ASIC consists of 25 channels (Fig. 13). In a typical configuration, 24 channels are configured for EEG measurements and 1 channel is configured as ECG channel. Each channel of the ASIC consists of a high CMRR instrumentation amplifier, followed by a variable gain amplifier. There are 8 different gain modes ranging from 200 to 10000 for the EEG channels and from 20 to 1000 for the ECG channel. The front end instrumentation amplifier has bandpass filter characteristics, where in-band gain and the cut-off frequencies are settable with external components. With an external capacitor of $1 \mu \mathrm{F}$, a bandwidth of $0.5-80 \mathrm{~Hz}$ is selected. The CMRR is larger than $90 \mathrm{~dB}$ at $50 \mathrm{mV}$ electrode offset. The total input referred voltage noise of each channel is less than $1 \mu \mathrm{Vrms}$ in the $0.5 \mathrm{~Hz}-80 \mathrm{~Hz}$ bandwidth. These features allow suppressing the input common mode voltages coupled to the human body, while amplifying the microvolt level biopotential signals. The mixed signal ASIC is designed and fabricated in $0.5 \mu \mathrm{m}$ CMOS process. The ASIC can operate from a voltage supply ranging from $2.7 \mathrm{~V}-3.3 \mathrm{~V}$ while dissipating less than $10.5 \mathrm{~mW}$. All the channels of the ASIC are multiplexed with a frequency of $1 \mathrm{kHz}$ per channel and buffered at the output. Therefore, a single ADC with a maximum input capacitance of $50 \mathrm{pF}$ can sample all the channels of the ASIC.

In a test setup, two channels of the $\mathrm{ASIC}$ are connected to $\mathrm{Ag} / \mathrm{AgCl}$ electrodes for reading the brain activity at the occipital cortex (backside of the head). A microcontroller with integrated ADC, is directly connected to the ASIC. Operation and gain settings of the ASIC are controlled from the microcontroller. When the patient closes his eyes, the typical alpha rhythm becomes clearly visible at the output (Fig. 14). 


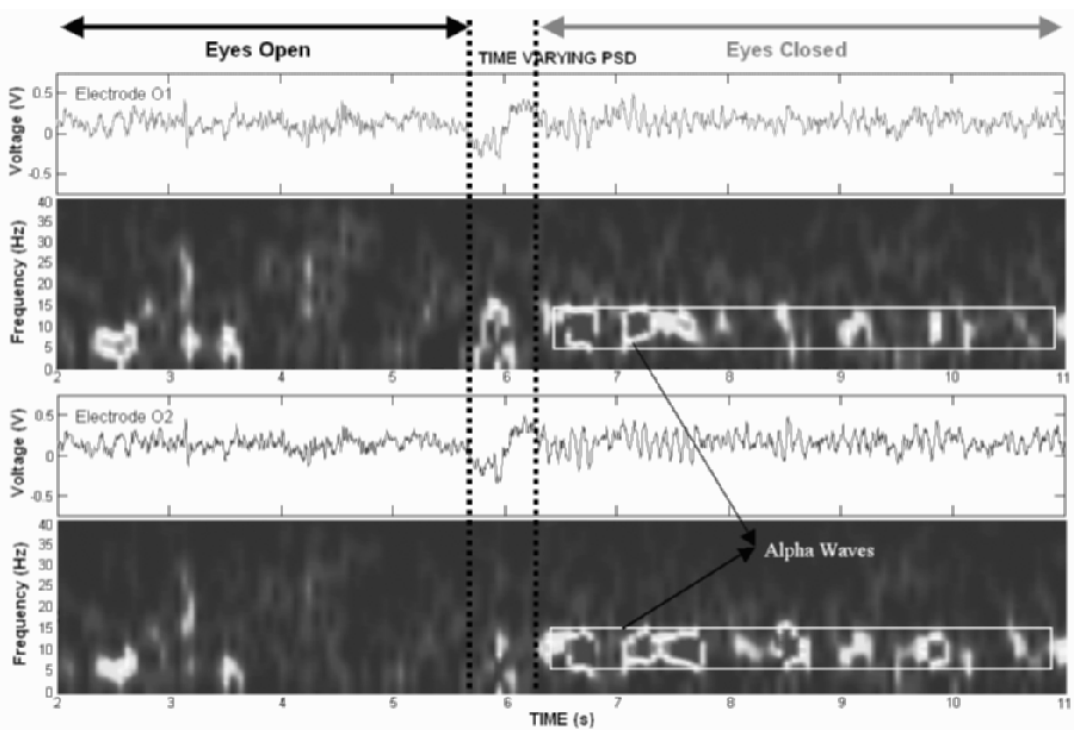

Fig. 14. Alpha activity from the two electrodes at occipital cortex, and their Short-Time Fourier Transform.

More recently we have also fabricated an alternative instrumentation amplifier architecture with a power consumption of $0.06 \mathrm{~mW}$ per channel while maintaining a CMRR of $120 \mathrm{~dB}$ [31]. This ASIC provides an additional factor of 20 in power savings per channel compared to the 24 channel version.

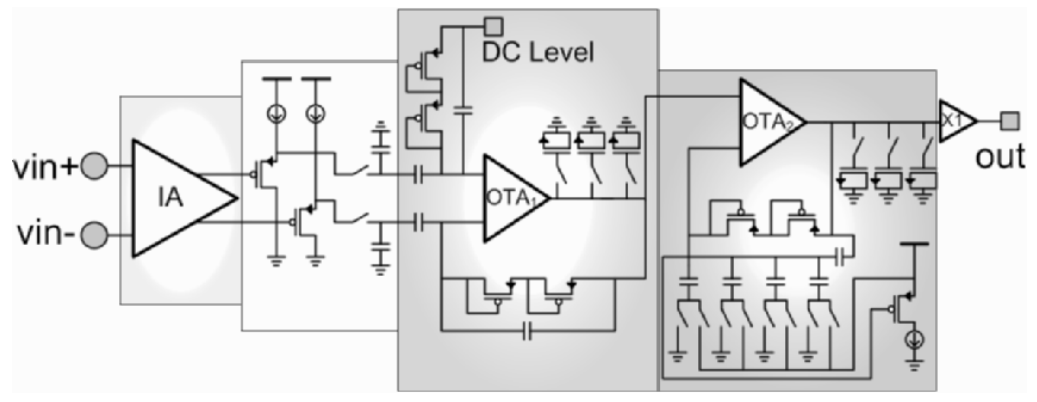

Fig. 15. Architecture of single channel biopotential ASIC.

\section{Integration Technology}

One form factor suitable for many sensor applications is a small cubic sensor node. To this end, a prototype wireless sensor node has been integrated in a cubic centimeter (Fig. 16). In this so-called three-dimensional system-in-a-package approach (3D SIP) [19], the different functional components are designed on separate boards and afterwards stacked on top of each other through a dual row of $0.7 \mathrm{~mm}$ solder balls with a pitch of $1.27 \mathrm{~mm}$. This system has the following advantages: 
(i) modules can be tested separately, (ii) functional layers can be added or exchanged depending on the application, (iii) each layer can be developed in the most appropriate technology. The first generation 3D stack offers a complete System-in-aPackage (SiP) solution for low power intelligent wireless communication. The first generation 3D stack offers a complete System-in-a-Package (SiP) solution for low power intelligent wireless communication. The integrated stack includes a commercial low power 8 MIPS microcontroller [20] and 2.4GHz transceiver [21], crystals and all necessary passives, as well as a matched dipole antenna custom-designed on the top layer laminate substrate. The bottom layer has a BGA footprint, allowing standard techniques for module mounting. This sensor module has been integrated with the watch-like thermal scavenger and consists in a basis for sensor networks [29], which, unlike most of their predecessors, are fully energy autonomous.

\section{Conclusion}

This text gave an overview of the Human++ research program, which is targeted at developing key technologies and components for future wireless body area networks for health monitoring applications. Several enabling technologies and integrated modules were discussed.

Over the next years, we will see more BAN technologies being developed all over the world. Step by step these will bring us closer to the end goal: an unobtrusive portable system that keeps track of our health and fitness level at an affordable cost.

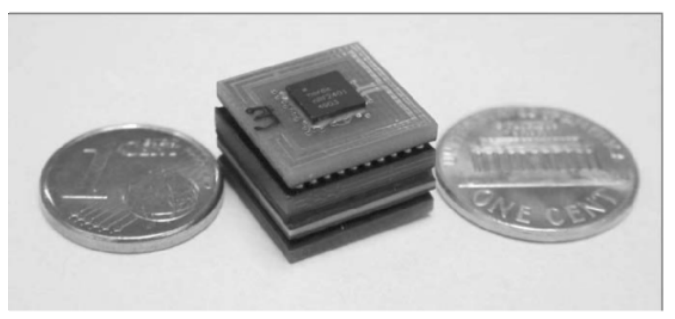

Fig. 16. 3D SiP Wireless autonomous sensor node.

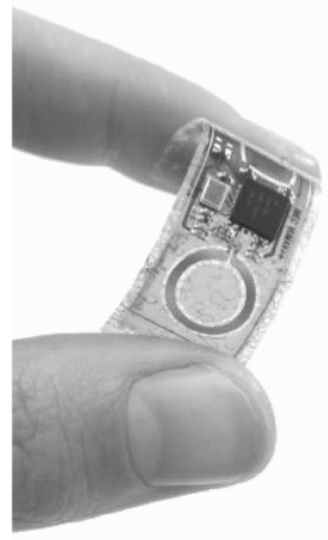

Fig. 17. Sensor in a flexible band aid.

Parallel research was started to implement the same technology on a flexible carrier. The ultimate target is to create a small and smart band-aid containing all the necessary technology for sensing and communication with a base station. It will provide a generic platform for various types of applications (wound healing, EEG, ECG, EMG...). The first prototype (Fig. 17) is 10 times smaller than a credit card 
$(12 \times 35 \mathrm{~mm})$ and about as thin as a compact disc $(1-2 \mathrm{~mm})$. The flexible $25 \mu \mathrm{m}$ polyimide carrier contains a microprocessor and a wireless communication module (2.4 GHz radio). It enables us to optimize the antenna for its activity on human skin. Current focus lies on adding the necessary sensors and energy equipment (rechargeable battery, energy scavenger and advanced electronics to keep energy consumption as low as possible). We target an ultimate device thickness of approximately $100 \mu \mathrm{m}$.

The biggest challenges in developing this kind of modules are the extreme miniaturization and its effects on the functionality of the used components. Some of the many problems to tackle are the use of naked chips, chip scaling, assembly processes like wire bonding and flip-chip on a flexible substrate, application of thinfilm batteries and solar cells and integration of the entire technology in a biocompatible package.

\section{References}

[1] http://www.imec.be/ovinter/static_research/human++.shtml

[2] R. Schmidt et al., "Body Area Network BAN, a key infrastructure element for patientcentered medical applications”, Biomed Tech (Berl). 2002;47 suppl 1 pt 1:365-8

[3] http://www.bluetooth.com, http://www.zigbee.org

[4] http://www.bluetooth.com/Bluetooth/Learn/Technology/Compare/Technical/

[5] IEEE 802.15.4a: http://www.ieee802.org/15/pub/tg4a.html

[6] http://www.rfm.com/products/data/tr1001.pdf

[7] B.W. Cook et al., "An ultra-low power $900 \mathrm{MHz}$ RF Transceiver for wireless sensor networks in 0.13 um cmos with $400 \mathrm{mV}$ supply and an integrated passive RX front-end", ISSCC, 2006, pp. 1460-1461

[8] Y.H. Chee, A.M. Niknejad, and J.M. Rabaey, "An ultra-low power MEMS-based twochannel transceiver for wireless sensor networks," Symposium on VLSI Circuits, June 2004, pp. 20-23

[9] M.Z. Win and R.A. Scholtz. Impulse radio: how it works. In IEEE Communications Letters, pp. 36-38, February 1998

[10] Federal Communications Commission (FCC). Revision of part 15 regarding ultrawideband transmission systems. First Report and Order, ET Docket, 98-153, FCC 02-48, adopted Feb. 2002, released Apr. 2002, available at http://www.fcc.gov

[11] http://www.ecma-international.org/publications/standards/Ecma-368.htm

[12] standard draft proposal. IEEE 802.15.4a available at http://www.ieee802.org/15/pub/ TG4a.html

[13] Y.H. Choi. Gated UWB pulse signal generation. In IEEE Joint International Workshop of UWBST and IWUWBS, pp. 122-124, May 2004

[14] J. Ryckaert, M. Badaroglu, C. Desset, V. de Heyn, G. Van der Plas, P. Wambacq, B. Van Poucke, and S. Donnay. Carrier-based uwb impulse radio: Simplicity, flexibility, and pulser implementation in $0.18 \mu \mathrm{m}$ cmos. International Conference on Ultrawideband, ICU 2005,2005

[15] R. Blazquez, F.S. Lee, D.D. Wentzloff, B. Ginsburg, J. Powell, and A.P. Chandrakasan. Direct conversion pulsed uwb transceiver architecture. Proc. of Design, Automation and Test in Europe, March 2005

[16] J. Ryckaert, M. Badaroglu, V. De Heyn, G. Van der Plas, P. Nuzzo, A. Baschirotto, S. D'Amico, C. Desset, H. Suys, M. Libois, B. Van Poucke, P. Wambacq, and B. Gyselinckx. A 16mA UWB 3-to-5Ghz 20Mpulses/s quadrature analog correlation 
receiver in $0.18 \mu \mathrm{m}$ CMOS. In ISSCC Digest of Technical Papers, pp. 114-115, February 2006

[17] J. Ryckaert, P. De Doncker, R. Meys, A. de Le Hoye, and S. Donnay, "Channel model for wireless communication around the human body", Electronics Letters, Vol. 40, Nr. 9, pp. 543-544, April 2004

[18] M. Verhelst, W. Vereecken, M. Steyaert, and W. Dehaene. Architectures for low power ultra-wideband radio receivers in the $3.1-5 \mathrm{GHz}$ band for data rates $<10 \mathrm{Mbps}$. In ISLPED'04, International Symposium on Low Power Electronics and Design, pp. 280285, August 2004

[19] S. Stoukatch, M. Ho, K. Vaesen, T. Webers, G. Carchon, W. De Raedt, E. Beyne, and J. De Baets, "Miniaturization using 3-D stack structure for SIP application", Proc. SMTA (Surface Mount Technology Association) International Conference, 21-29 September 2003; Chicago.

[20] TI MSP430F149, www.ti.com

[21] Nordic nRF2401, www.nvlsi.com

[22] V. Leonov, P. Fiorini, S. Sedky, T. Torfs, and C. Van Hoof, "Thermoelectric MEMS generators as a power supply for a body area network". Proc. 13-th Int. Conf. Solid-State Sensors, Actuators and Microsystems (Transducers'05), Seoul, Korea, June 5-9, 2005, pp. 291-294

[23] V. Leonov, T. Torfs, P. Fiorini, and C. Van Hoof, "Thermoelectric converters of human warmth for self-powered wireless sensor nodes". IEEE Sensors Journal, 2007 (in press)

[24] T. Torfs, V. Leonov, B. Gyselinckx, and C. Van Hoof, "Body-Heat Powered Autonomous Pulse Oximeter", proceedings, IEEE Sensors, 2006, Korea, in press

[25] S.J. Roundy, "Energy Scavenging for Wireless Sensor Nodes with a Focus on Vibration to Electricity Conversion", PhD Thesis, UC Berkeley, 2003

[26] B. Köhler, H. Carsten, and R. Orglmeister: The Principles of Software QRS Detection, Feb. 2002, IEEE Engineering in Medicine and Biology, 42-57

[27] T.R. Halfhill: Silicon Hive Breaks Out, Dec. $1^{\text {st }}$ 2003, Microprocessor Report, www.MPRonline.com

[28] J. Pan and W.J. Tompkins: A Real-Time QRS Detection Algorithm, 1985, IEEE Transactions Biomedical Engineering, BME-32(3): 230-236

[29] M. Tubasihat and S. Madria, "Sensor networks: an overview", IEEE Potentials, 2003, Vol. 22(2), 20-23

[30] R.F. Yazicioglu, P. Merken, and C. Van Hoof, "Integrated Low-Power 24-Channel EEG Front-End”, IEE Electronics Letters, Vol. 41, iss. 8, pp. 457-458, Apr. 2005

[31] R.F. Yazicioglu, P. Merken, R. Puers, and C. Van Hoof, "A $60 \mu \mathrm{W} 60 \mathrm{nV} / \sqrt{ } \mathrm{Hz}$ Readout Front-End for Portable Biopotential Acquisition Systems," IEEE J. Solid-State Circuits, 2007

[32] A.A. Ahmed, "A Survey on Network Protocols for Wireless Sensor Networks", Information Technology: Research and Education, 2003 proceedings, pp. 301-305

[33] M.A.M. Vieira et al., "Survey on wireless sensor network devices", IEEE Emerging Technologies and Factory Automation 2003 proceedings, Vol. 1, pp. 537-544

[34] B.A. Warneke et al., "An autonomous $16 \mathrm{~mm}^{3}$ solarpowered node for distributed wireless sensor networks", IEEE Sensors 2002 proceedings, Vol. 2, pp. 1510-1515

[35] T. Torfs, S. Sanders, C. Winters, S. Brebels, and C. Van Hoof, "Wireless network of autonomous environmental sensors", Proceedings of IEEE Sensors 2004, Vienna, 24-27 October 2004. 